\title{
Relationship between abnormal glucose metabolism and osteoporosis in Han Chinese men over the age of 50 years
}

\author{
Minyan Liu \\ Yanhui Lu \\ Xiaoling Cheng \\ Lichao Ma \\ Xinyu Miao \\ Nan Li \\ Boruo Sun \\ Shuangtong Yan \\ Jian Li \\ Chunling Li \\ Department of Elderly Endocrinology, \\ Chinese PLA General Hospital, \\ Haidian District, Beijing I00853, China
}

Correspondence: Chunling Li Department of Elderly Endocrinology, Chinese PLA General Hospital, No 28, Fuxing Road, Haidian District, Beijing 100853, China

$\mathrm{Tel}+86$ I50 I | 35 030 I

Email Iclin30I@I26.com
This article was published in the following Dove Medical Press journal:

Clinical Interventions in Aging

Aim: The aim of this study was to determine the relationship between abnormal glucose metabolism and osteoporosis (OP) in Han Chinese men over the age of 50 years.

Patients and methods: A cross-sectional study of 775 male patients aged over 50 years was performed at our hospital in 2011. The patients were divided into a normal glucose metabolism group, an impaired glucose regulation (IGR) group, and a type 2 diabetes mellitus (T2DM) group. Differences in their bone mineral densities (BMDs), OP detection rates, and indices of bone metabolism were assessed.

Results: After adjusting for age and body mass index (BMI), there were no significant differences in lumbar spine, femoral neck, and total hip BMD values in the three groups $(P>0.05)$ nor in OP detection rates $(P=0.19)$. However, there were some significant differences in bone metabolism markers between the groups after adjusting for age, BMI, and serum creatinine (Cr): 25-hydroxyvitamin $\mathrm{D}(25(\mathrm{OH}) \mathrm{D})$ was positively correlated with the presence of abnormal glycometabolism $(r=0.08 ; P<0.01)$, while $\beta$-carboxy-terminal cross-linking telopeptide of type I collagen ( $\beta$-CTX), bone gamma-carboxyglutamic acid protein (BGP; osteocalcin $[\mathrm{OC}]$ ), and procollagen type 1 intact $\mathrm{N}$-terminal propeptide (P1NP) were negatively correlated ( $r=-0.13,-0.21,-0.14$, respectively; $P<0.01$ ). Logistic regression analysis of the data indicated that BGP was the only bone metabolism marker significantly influenced by abnormal glucose metabolism $(\mathrm{OR}=0.96)$.

Conclusion: There were no significant differences in BMD or OP detection rates between the three glycometabolism groups after adjusting for age and BMI. However, the bone metabolism marker, BGP, was significantly negatively correlated with abnormal glucose metabolism.

Keywords: diabetes mellitus, osteoporosis, bone mineral density, bone turnover markers

\section{Introduction}

Diabetes mellitus (DM) is a major chronic disease especially among people above middle age. About one in five people above the age of 50 years in China would have DM. ${ }^{1}$ However, the main reasons for death and disability among DM patients are macroangiopathy and microangiopathy. But recently, studies have shown that DM, both type $1 \mathrm{DM}$ and type $2 \mathrm{DM}$ (T2DM), has significantly increased risk of vertebral, hip, and all nonvertebral fractures. ${ }^{2}$ Osteoporosis (OP) is an age-related disease characterized as lower bone mineral density (BMD) and higher risk of fracture. One-third of the population above the age of 50 years suffer from OP in China. ${ }^{3}$

BMD is a diagnostic index of OP. The association between DM and BMD has atracted a lot of researchers. In addition, type $1 \mathrm{DM}$ is known to lead to a decline in BMD and an increased fracture risk, but differing results have been reported for T2DM. 
A previous meta-analysis showed that, in 47 studies, most studies showed an increased BMD in DM patients, 13 showed a decreased BMD, and eight showed a normal BMD or no difference in BMD. ${ }^{4}$ However, there were only two studies of Chinese included. Because BMD was affected by ethnic differences and corresponding living habits and hormone levels, ${ }^{5}$ there is still a gap for Han Chinese people of the relationship between T2DM and BMD. In addition, data on the relationship between abnormal glucose metabolism and BMD and bone turnover markers (BTMs) are lacking. The aim of this study was to determine the relationships between abnormal glucose metabolism and OP and assess BMD and BTM values in Han Chinese men over the age of 50 years.

\section{Patients and methods}

\section{Patients}

A cross-sectional study of 775 Han Chinese men over the age of 50 years was performed at our hospital in Beijing in 2011. The patients were divided into three groups on the basis of their glucose metabolism status: a normal group; an impaired glucose regulation (IGR) group (which included patients with diagnoses of impaired fasting glucose [IFG] and impaired glucose tolerance [IGT]); and a T2DM group. In addition, they were divided into three groups on the basis of WHO osteoporosis diagnostic criteria, ie, normal, osteopenia, and OP groups.

Inclusion criteria for the study were as follows: 1) men aged 50 years or older; 2) the Han nationality; 3) who completed routine physical examination and bone density detection.

Exclusion criteria for the study were as follows: 1) other metabolic diseases including delayed puberty, low sex hormone levels, hyperparathyroidism, and hyperthyroidism; 2) COPD; 3) the presence of bone tumors; 4) treatment with drugs that influence bone metabolism (including corticosteroids and gonadotropin-releasing hormone drugs); 5) treatment with drugs for OP (including bisphosphonates, calcitonin, strontium ranelate, and teriparatide); 6) the presence of severe liver disease and kidney disease; 7) excessive alcohol ingestion or smoking; 8) patients who had been non-ambulant for more than 3 months prior to the study; and 9) patients who had undergone bilateral hip fracture or hip replacement surgery.

All eligible subjects were asked to provide written informed consent to participate in the study. All participants had the right to reject this study or withdraw from this study anytime. Approval for the study was provided by the ethics committee of the General Hospital of the People's Liberation Army, Beijing, China.

\section{Data collection and testing methods}

The patients' histories were taken, and routine physical examinations were performed by the same trained health care personnel. Subjects were requested to undergo an oral glucose tolerance test (OGTT) at the same time, for which the blood was drawn at baseline and 2 hours later. In addition, tests were performed for biochemical markers (liver and kidney function parameters, uric acid, serum lipids, calcium, phosphorus, ALP), bone metabolism markers such as parathyroid hormone (PTH) and 25-hydroxyvitamin D (25(OH)D), and BTMs such as bone gamma-carboxyglutamic acid protein (BGP; osteocalcin [OC]), procollagen type 1 intact $\mathrm{N}$-terminal propeptide (P1NP), and $\beta$-carboxy-terminal cross-linking telopeptide of type I collagen ( $\beta$-CTX).

\section{Testing bone metabolism and BTMs}

For testing bone metabolism and BTMs, $3 \mathrm{~mL}$ of blood samples was drawn and serum was stored at $-80^{\circ} \mathrm{C}$ after cryogenic centrifugal separation. Concentrations of $25(\mathrm{OH}) \mathrm{D}$, PTH, BGP, P1NP, and $\beta$-CTX were measured by an automatic electrochemical luminescence method (Roche Cobas E 601 [coefficient of variation $<10 \%$ ]). This technique measures $25(\mathrm{OH}) \mathrm{D}$ concentrations in the range of 4-100 ng/ $\mathrm{mL}$; PTH concentrations in the range of $20-5,000 \mathrm{pg} / \mathrm{mL}$; BGP concentrations in the range of $0.5-300 \mathrm{ng} / \mathrm{mL}$; P1NP concentrations in the range of $5-1,200 \mathrm{ng} / \mathrm{mL}$; and $\beta$-CTX concentrations in the range of $0.01-6.0 \mathrm{ng} / \mathrm{mL}$.

\section{BMD estimators}

All BMD measurements were made by dual-energy X-ray absorptiometry (DXA) scans (GE-UNAR Company, Boston, MA, USA [coefficient of variation $<1.2 \%$ ]) which were performed by the same trained operator. Patients were asked to remove all metal objects from their bodies and clothes (eg, metal zippers and metal buttons) prior to undergo DXA scans, and it was ascertained that they had not been exposed to radionuclides or imaging agents within the last 3 days prior to scanning. Sites at which BMD measurements were made were the proximal femurs (total hip, femoral neck) and the lumbar spine 1-4 (L1-4). Patients were positioned in a lying position with their legs turned slightly inward and held in place by the use of positioning devices.

\section{Diagnostic criteria}

- OP: by utilizing the 1994 WHO diagnostic criteria, patients were classified as either being normal or having osteopenia or OP according to $\mathrm{T}$ score. Normal bone mass was defined as $\mathrm{T}$ score $>-1$. Osteopenia was defined as $\mathrm{T}$ score $>-2.5$ and $\leq-1$ and OP was defined 
as $\mathrm{T}$ score $\leq-2.5$. The lowest $\mathrm{T}$ scores of the total hip, femur neck, or lumbar spine (L1-4) were used for the diagnosis of OP or osteopenia.

- Diabetes and related metabolic diseases: by utilizing the 1999 WHO diagnostic criteria for impaired glucose metabolism, patients were classified as having either: 1) normal glucose tolerance; 2) IGR, which included patients with either IFG (fasting plasma glucose [FPG] 6.1-6.9 mmol/L [110-125 mg/dL] and 2-hour postprandial glucose [2-hour PPG] $<7.8 \mathrm{mmol} / \mathrm{L}$ [140 mg/dL]) or IGT (FPG 3.9-6.1 mmol/L [70-110 mg/dL] and 2-hour PPG 7.8-11.0 mmol/L [140-200 mg/dL]); or 3) $\mathrm{T} 2 \mathrm{DM}(\mathrm{FPG}>7.0 \mathrm{mmol} / \mathrm{L}[126 \mathrm{mg} / \mathrm{dL}]$ and/or 2 -hour PPG $\geq 11.1 \mathrm{mmol} / \mathrm{L}$ [200 mg/dL]). Blood glucose concentrations were measured using venous blood, and the results were confirmed by repeating the measurements at least once. Patients with DM include both the selfreported patients with a definitive doctor's diagnosis and the newly diagnosed patients based on the OGTT result.

- Metabolic syndrome (MS): by utilizing the 2004 Chinese Diabetes Society (CDS) criteria for MS, patients were classified as either 1) overweight or obese (body mass index $[\mathrm{BMI}] \geq 25 \mathrm{~kg} / \mathrm{m}^{2}$ ); 2) hypertensive (SBP $\geq 140 \mathrm{mmHg}$ and/or DBP $\geq 90 \mathrm{mmHg}$, or a previous diagnosis of hypertension); 3) dyslipidemic (triglycerides [TGs] $\geq 150 \mathrm{mg} / \mathrm{dL}[1.7 \mathrm{mmol} / \mathrm{L}]$, highdensity lipoprotein cholesterol [HDL-C] $<35 \mathrm{mg} / \mathrm{dL}$ [0.9 mmol/L], or a previous diagnosis of dyslipidemia); or 4) hyperglycemic (FPG $\geq 110 \mathrm{mg} / \mathrm{dL}[6.1 \mathrm{mmol} / \mathrm{L}]$ and/or 2-hour PPG $\geq 140 \mathrm{mg} / \mathrm{dL}[7.8 \mathrm{mmol} / \mathrm{L}]$, or a previous diagnosis of DM).

- Vitamin D nutritional status: the serum concentration of $25(\mathrm{OH}) \mathrm{D}$ was used to classify the patients' vitamin $\mathrm{D}$ status as either severely deficient $(25(\mathrm{OH})$ $\mathrm{D}<10 \mathrm{ng} / \mathrm{mL})$; deficient (25(OH)D 10-20 ng/mL); insufficient ( $20 \mathrm{ng} / \mathrm{mL} \leq 25(\mathrm{OH}) \mathrm{D}<30 \mathrm{ng} / \mathrm{mL})$; or sufficient $(25(\mathrm{OH}) \mathrm{D} \geq 30 \mathrm{ng} / \mathrm{mL})$. A serum $25(\mathrm{OH}) \mathrm{D}$ concentration of $<30 \mathrm{ng} / \mathrm{mL}$ was defined as a low vitamin $\mathrm{D}$ level.

\section{Statistical analyses}

Statistical analyses were performed using STATA10.0 software (StataCorp LP, College Station, TX, USA). All data are presented as mean $\pm \mathrm{SD}$. Continuous variables with a normal distribution were analyzed by univariate ANOVA and adjusted analysis of covariance (ANCOVA) for adjusting associated factors. Comparisons between different groups were analyzed by chi-squared tests with data expressed in percentage terms. Linear correlation analysis was also carried out using the Pearson correlation followed by stepwise multiple logistic regression analysis. For the glucometabolic and osteoporotic groups, Spearman's rank correlation coefficient was used. For all comparisons, statistical significance was defined as a $P$-value of $<0.05$.

\section{Results \\ General characteristics of the glycometabolism groups}

A comparison of the general characteristics of the three glycometabolism groups (normal [ $\mathrm{n}=142]$, IGR [n=245], and DM [ $n=388]$; Table 1) showed that age, SBP, FPG, PPG, TG, $\mathrm{HbA1c}$, and serum creatinine $(\mathrm{Cr})$ tended to increase with increasing glucose intolerance $(P<0.05)$, whereas weight, DBP, total cholesterol (TC), HDL-C, and LDL-C decreased $(P<0.05)$. After adjusting for age, there were still statistically significant differences between the groups for FPG, PPG, HbA1c, TC, TG, HDL-C, LDL-C, and Cr (Table 1). However, differences between the groups for other characteristics were not statistically significant.

\section{Relationship between abnormal glycometabolism and metabolic disease characteristics}

The percentages of patients with dyslipidemia and MS in the three glycometabolism groups were higher in the DM group (47.09\% and $56.35 \%$, respectively) than in the IGR group (29.38\% and $52.13 \%$, respectively) and the normal glucose tolerance group $(29.03 \%$ and $12.90 \%$, respectively; $P<0.01)$. However, for patients classified as overweight or obese, there was no significant difference between the three glycometabolism groups $(41.53 \%, 39.34 \%$, and $31.45 \%$, respectively, in the DM, IGR, and normal groups; $P=0.14$ ).

\section{Relationship between abnormal glycometabolism and BMD characteristics} Statistically significant differences in BMD were found among the three glycometabolism groups (Table 2). BMD values in the hip were lowest in the IGR group, while lumbar BMD values were highest in the IGR group. However, after adjusting for age and BMI, there were no statistically significant differences in BMD values between the glycometabolism groups ( $P>0.05$; Table 2$)$.

\section{Relationship between abnormal glycometabolism and OP and osteopenia detection rates}

The OP detection rate in the DM group was lower than that in the normal and IGR groups. The highest OP detection rate was observed in the IGR group (15.10\%), and this was 
Table I General characteristics of the three glycometabolism groups

\begin{tabular}{|c|c|c|c|c|c|c|c|}
\hline Characteristics & Normal $(n=\mid 42)$ & IGR (n=245) & T2DM $(n=388)$ & $F$-value & $P$-value & $\begin{array}{l}\text { F-value } \\
\text { adjusted } \\
\text { for age }\end{array}$ & $\begin{array}{l}P \text {-value } \\
\text { adjusted } \\
\text { for age }\end{array}$ \\
\hline Age, years & $73.5 \pm 11.0$ & $75.7 \pm I I . I$ & $76.7 \pm 10.8$ & 5.00 & 0.00 & & \\
\hline Height, cm & $171.13 \pm 4.88$ & $170.12 \pm 5.44$ & $170.3 \pm 5.90$ & 1.71 & 0.18 & & \\
\hline Weight, kg & $73.35 \pm 10.26$ & $72.4 \pm 10.27$ & $71.08 \pm 10.34$ & 6.81 & 0.00 & 2.13 & 0.12 \\
\hline $\mathrm{BMI}, \mathrm{kg} / \mathrm{m}^{2}$ & $24.0 \pm 2.81$ & $24.32 \pm 2.82$ & $24.45 \pm 3.03$ & 1.43 & 0.24 & 2.82 & 0.06 \\
\hline $\mathrm{SBP}, \mathrm{mmHg}$ & $129.73 \pm 18.62$ & $|29.15 \pm| 6.7 \mid$ & $132.31 \pm 16.75$ & 6.89 & 0.00 & 2.67 & 0.07 \\
\hline $\mathrm{DBP}, \mathrm{mmHg}$ & $77.10 \pm 10.87$ & $74.37 \pm 9.96$ & $72.75 \pm 10.72$ & 5.23 & 0.00 & 0.39 & 0.68 \\
\hline FPG, $\mathrm{mmol} / \mathrm{L}$ & $5.17 \pm 0.38$ & $5.44 \pm 0.50$ & $6.38 \pm 1.60$ & 9.23 & 0.00 & 8.90 & 0.00 \\
\hline PPG, $\mathrm{mmol} / \mathrm{L}$ & $5.92 \pm 0.76$ & $8.23 \pm 1.28$ & $11.13 \pm 2.90$ & 5.87 & 0.00 & 6.48 & 0.00 \\
\hline $\mathrm{FCP}, \mathrm{ng} / \mathrm{mL}$ & $2.34 \pm 1.18$ & $2.6 \mathrm{I} \pm \mathrm{I} .1 \mathrm{I}$ & $2.42 \pm 0.82$ & 1.05 & 0.35 & & \\
\hline FINS, mU/L & $7.62 \pm 5.03$ & $8.55 \pm 4.86$ & $8.16 \pm 5.44$ & 0.40 & 0.67 & & \\
\hline $\mathrm{HbAlc}$ \% (mmol/mol) & $5.77 \pm 0.36(40 \pm 3.9)$ & $6.30 \pm 0.94(45 \pm 10.3)$ & $6.59 \pm 1.01(49 \pm 11.0)$ & 6.10 & 0.00 & 3.60 & 0.03 \\
\hline $\mathrm{TC}, \mathrm{mmol} / \mathrm{L}$ & $4.86 \pm 0.90$ & $4.7 I \pm 0.95$ & $4.5 \pm 0.95$ & 9.43 & 0.00 & 6.33 & 0.00 \\
\hline $\mathrm{TG}, \mathrm{mmol} / \mathrm{L}$ & $1.43 \pm 0.82$ & $\mid .47 \pm 0.9$ | & $1.48 \pm 0.92$ & 5.06 & 0.00 & 8.58 & 0.00 \\
\hline HDL-C, $\mathrm{mmol} / \mathrm{L}$ & $1.33 \pm 0.33$ & $\mathrm{I} .3 \mathrm{I} \pm 0.34$ & $1.27 \pm 0.35$ & 5.82 & 0.00 & 6.88 & 0.00 \\
\hline LDL-C, $\mathrm{mmol} / \mathrm{L}$ & $3.08 \pm 0.8 I$ & $2.98 \pm 0.81$ & $2.82 \pm 0.84$ & 6.27 & 0.00 & 8.35 & 0.00 \\
\hline Serum $\mathrm{Cr}, \mu \mathrm{mol} / \mathrm{L}$ & $85.23 \pm 14.54$ & $86.38 \pm 18.78$ & $87.19 \pm 21.97$ & 5.94 & 0.00 & 5.75 & 0.00 \\
\hline $\mathrm{ALT}, \mathrm{U} / \mathrm{L}$ & $17.60 \pm 10.79$ & $|9.67 \pm||.2|$ & $20.28 \pm 11.75$ & 2.83 & 0.06 & & \\
\hline $\mathrm{UA}, \mu \mathrm{mol} / \mathrm{L}$ & $353.58 \pm 69.55$ & $356 \pm 75.15$ & $350.68 \pm 79.57$ & 1.00 & 0.37 & & \\
\hline
\end{tabular}

Notes: Values are presented as mean \pm SD. ANOVA was used for analysis.

Abbreviations: ALT, alanine aminotransferase; BMI, body mass index; Cr, creatinine; FCP, fasting C-peptide; FINS, fasting serum insulin; FPG, fasting plasma glucose; HDL-C, high-density lipoprotein cholesterol; IGR, impaired glucose regulation; LDL-C, low-density lipoprotein cholesterol; PPG, postprandial glucose; T2DM, type 2 diabetes mellitus; TC, total cholesterol; TG, triglyceride; UA, uric acid.

significantly higher than that in the other glycometabolism groups ( $P=0.03$; Table 3$)$.

Spearman's correlation analysis indicated that OP detection was negatively correlated with abnormal glucose metabolism $(r=-0.10 ; P<0.01)$. However, after adjusting for age and BMI, a rank transformation test showed that there were no statistically significant differences between the groups $(P=0.19)$.

\section{Relationship between abnormal glycometabolism and bone metabolism markers}

Concentrations of calcium $(\mathrm{Ca})$, phosphorus $(\mathrm{P}), 25(\mathrm{OH}) \mathrm{D}$, PTH, $\beta$-CTX, P1NP, and BGP were significantly different in the three glycometabolism groups (Table 4). After adjusting for age, $\mathrm{Cr}$, and $\mathrm{BMI}$, there were still statistically significant differences for $\mathrm{Ca}, \mathrm{P}, 25(\mathrm{OH}) \mathrm{D}, \beta-\mathrm{CTX}$, BGP, and P1NP (Table 4).

Spearman's correlation analysis was performed to analyze the correlation between bone metabolism markers and abnormal glucose metabolism. The results showed that abnormal glucose metabolism was positively correlated with 25(OH)D $(r=0.08 ; P<0.01)$, but negatively correlated with $\beta$-CTX, BGP, and P1NP ( $r=-0.13, r=-0.21$ and $r=-0.14$, respectively; all $P<0.01)$.

Multiple logistic regression analysis was performed with 25(OH)D, $\beta$-CTX, BGP, P1NP, Cr, age, BMI, and weight as categorical independent variables and the glycometabolism

Table 2 BMD values in different parts of the body in the three glycometabolism groups

\begin{tabular}{|c|c|c|c|c|c|c|c|c|}
\hline \multirow[t]{2}{*}{ Group } & \multirow{2}{*}{$\begin{array}{l}\text { No of } \\
\text { patients }\end{array}$} & \multicolumn{7}{|c|}{ BMD $\left(\mathrm{g} / \mathrm{cm}^{2}\right)$} \\
\hline & & LI-4 & FN-R & FN-L & Troch-R & Troch-L & Total-R & Total-L \\
\hline Normal & 142 & $1.189 \pm 0.197$ & $0.866 \pm 0.148$ & $0.876 \pm 0.138$ & $0.803 \pm 0.132$ & $0.817 \pm 0.129$ & $0.955 \pm 0.149$ & $0.964 \pm 0.144$ \\
\hline IGR & 245 & $1.214 \pm 0.221$ & $0.855 \pm 0.142$ & $0.859 \pm 0.140$ & $0.793 \pm 0.136$ & $0.806 \pm 0.138$ & $0.948 \pm 0.151$ & $0.956 \pm 0.152$ \\
\hline T2DM & 388 & $1.204 \pm 0.185$ & $0.884 \pm 0.155$ & $0.879 \pm 0.124$ & $0.815 \pm 0.118$ & $0.823 \pm 0.122$ & $0.976 \pm 0.127$ & $0.979 \pm 0.132$ \\
\hline$P$-value adjusted for age and BMI & & 0.61 & 0.59 & 0.54 & 0.39 & 0.76 & 0.19 & 0.46 \\
\hline$P$-value adjusted for age, & & 0.22 & 0.53 & 0.64 & 0.63 & 0.35 & 0.87 & 0.88 \\
\hline BMI, height, and weight & & & & & & & & \\
\hline
\end{tabular}

Notes: Data are expressed as mean \pm SD. Spearman's rho was used to detect the relationship between abnormal glycometabolism and OP and osteopenia detection rates. Abbreviations: BMD, bone mineral density; BMI, body mass index; FN, femoral neck; IGR, impaired glucose regulation; L, left; LI-4, lumbar vertebrae I-4; OP, osteoporosis; $\mathrm{R}$, right; T2DM, type 2 diabetes mellitus; Troch, trochanter. 
Table 3 Detection rates of OP and osteopenia in the three glycometabolism groups (n, \%)

\begin{tabular}{l|l|l|l|l}
\hline & Normal $(\mathbf{n}=\mathbf{I 4 2})$ & IGR $(\mathbf{n}=\mathbf{2 4 5})$ & T2DM $(\mathbf{n}=\mathbf{3 8 8})$ & $\boldsymbol{P}$-value \\
\hline Not detected & $6 I(4 I .96)$ & $10 I(4 I .22)$ & $189(48.7 I)$ & \\
Osteopenia & $66(46.48)$ & $107(43.67)$ & $170(43.8 I)$ & 0.03 \\
OP & $15(10.56)$ & $37(15.10)$ & $29(7.47)$ & \\
\hline
\end{tabular}

Note: Spearman's rho was used for analysis.

Abbreviations: IGR, impaired glucose regulation; OP, osteoporosis; T2DM, type 2 diabetes mellitus.

groups as dependent variables (Table 5). The results showed that BGP (OC) was the only bone metabolism marker to be significantly influenced by abnormal glucose metabolism ( $\mathrm{OR}=0.96)$.

Linear correlation analysis indicated that, while BGP was negatively correlated with FPG and PPG ( $r=-0.15$ and $r=-0.10$, respectively; $P<0.05$ ), fasting serum insulin (FINS) and fasting $\mathrm{C}$-peptide (FCP) were not correlated with $\mathrm{HbA1c}$.

\section{Discussion}

With the progressive aging of populations around the world, the prevalence of diabetes has increased dramatically in recent years such that diabetes and its complications are now responsible for a serious societal health burden. In 2013, an epidemiological survey of DM in China showed that the prevalence of diabetes was $11.6 \%$ in adult people and that $50.1 \%$ could be classified as prediabetic. Thus, three in five adults in China suffer from hyperglycemia. However, due to delays in diagnosis, $74.2 \%$ of Chinese patients with diabetes are not treated soon enough. ${ }^{1}$

Albright ${ }^{6}$ reported for the first time that the long-term poor glycemic control can lead to OP. Since this report, the relationship between diabetes and osteoporotic fractures has attracted widespread attention by researchers, and diabetic osteopathy is now recognized as one of the most important chronic complications of diabetes. The prevalence of both diabetes and OP is high, and they increase with increasing age. Common causes of death and disability in elderly people with diabetes are microvascular disease and osteoporotic fractures.

Although type $1 \mathrm{DM}$ is known to lead to a reduction in BMD and an increased fracture risk, the relationship between T2DM and BMD is uncertain. Most studies suggest that BMD in patients with T2DM is normal or even increased and generally not decreased. However, other studies have shown that the prevalence of OP is increased in patients with diabetes and a low BMD in comparison with nondiabetic controls. $^{7-9}$ In 2012, a meta-analysis that systematically analyzed 15 observational studies of BMD in patients with T2DM (3,437 diabetics, 19,139 controls) found that BMD was significantly higher in patients with T2DM than in the nondiabetic controls. ${ }^{10} \mathrm{~A}$ younger age, male gender, a high BMI, and a high $\mathrm{HbAlc}$ value were positively correlated with BMD in diabetic individuals. The meta-analysis concluded that multiple factors influence BMD in patients with T2DM. ${ }^{9}$

After adjusting for age, blood glucose concentrations, $\mathrm{HbAlc}$, and $\mathrm{Cr}$ showed an increasing trend with increasing glucose intolerance in our study. As the $P$-value for BMI differences between the three glycometabolism groups was 0.06 after correction for age, it was concluded that for the analysis of BMD and bone metabolism markers, the covariance analysis needed corrections for age, BMI, and $\mathrm{Cr}$. In comparison with the normal glycometabolism group, there were no significant differences in BMD values in the lumbar

Table 4 Markers of bone metabolism and bone turnover in the three glycometabolism groups

\begin{tabular}{|c|c|c|c|c|c|c|c|}
\hline Marker & Normal & IGR & T2DM & F-value & $P$-value & $\begin{array}{l}\text { F-value adjusted } \\
\text { for serum } \mathrm{Cr} \text {, } \\
\text { age, } \mathrm{BMI}\end{array}$ & $\begin{array}{l}P \text {-value adjusted } \\
\text { for serum } \mathrm{Cr} \text {, } \\
\text { age, } \mathrm{BMI}\end{array}$ \\
\hline $\mathrm{Ca}, \mathrm{mmol} / \mathrm{L}$ & $2.36 \pm 0.08$ & $2.35 \pm 0.09$ & $2.36 \pm 0.09$ & 5.18 & 0.00 & 3.52 & 0.03 \\
\hline $\mathrm{P}, \mathrm{mmol} / \mathrm{L}$ & $1.09 \pm 0.15$ & $1.08 \pm 0.14$ & $I . I \pm 0.15$ & 2.89 & 0.06 & 3.94 & 0.02 \\
\hline ALP, U/L & $63.85 \pm 17.70$ & $62.83 \pm 16.22$ & $63.14 \pm 17.59$ & $|.3|$ & 0.27 & 1.51 & 0.23 \\
\hline $25(\mathrm{OH}) \mathrm{D}, \mathrm{ng} / \mathrm{mL}$ & $20.36 \pm 8.94$ & $21.82 \pm 9.30$ & $22.49 \pm 10.14$ & 5.33 & 0.00 & 4.63 & 0.01 \\
\hline PTH, pg/mL & $38.10 \pm 14.36$ & $41.09 \pm 15.97$ & $39.17 \pm 15.27$ & 8.21 & 0.00 & 1.72 & 0.18 \\
\hline$\beta$-CTX, ng/mL & $0.30 \pm 0.15$ & $0.29 \pm 0.16$ & $0.26 \pm 0.16$ & 6.40 & 0.00 & 3.84 & 0.02 \\
\hline BGP, ng/mL & $18.25 \pm 8.32$ & $16.56 \pm 7.85$ & $15.05 \pm 7.03$ & 5.34 & 0.00 & 7.75 & 0.00 \\
\hline PINP, ng/mL & $38.12 \pm 14.28$ & $35.85 \pm 17.45$ & $34.09 \pm 22.12$ & 10.24 & 0.00 & 3.79 & 0.02 \\
\hline
\end{tabular}

Notes: Values are presented as mean \pm SD. ANOVA was used for analysis.

Abbreviations: BGP, bone gamma-carboxyglutamic acid protein (osteocalcin); BMI, body mass index; Ca, calcium; Cr, creatinine; $\beta$-CTX, $\beta$-carboxy-terminal cross-linking telopeptide of type I collagen; 25(OH)D, 25-hydroxyvitamin D; IGR, impaired glucose regulation; P, phosphorus; PINP, procollagen type I intact N-terminal propeptide; PTH, parathyroid hormone; T2DM, type 2 diabetes mellitus. 
Table 5 Multiple logistic regression analysis for the glycometabolism groups

\begin{tabular}{l|l|l|l|l}
\hline & OR & z-value & $\boldsymbol{P}$-value & $\mathbf{9 5 \%} \mathbf{C l}$ \\
\hline Age & 1.07 & 10.78 & 0.00 & $1.06-1.09$ \\
BGP & 0.96 & -2.89 & 0.004 & $0.94-0.99$ \\
BMI & 1.06 & 2.61 & 0.009 & $1.02-1.12$ \\
Serum Cr & 0.99 & -2.09 & 0.037 & $0.99-1.0$ \\
\hline
\end{tabular}

Notes: $25(\mathrm{OH}) \mathrm{D}, \beta-\mathrm{CTX}, \mathrm{BGP}, \mathrm{PINP}, \mathrm{Cr}$, age, BMI, and weight as categorical independent variables and the glycometabolism groups as dependent variables. The Wald value is 225.38 .

Abbreviations: $\beta$-CTX, $\beta$-carboxy-terminal cross-linking telopeptide of type I collagen; BGP, bone gamma-carboxyglutamic acid protein (osteocalcin); BMl, body mass index; $\mathrm{Cr}$, creatinine; 25(OH)D, 25-hydroxyvitamin D; PINP, procollagen type I intact N-terminal propeptide.

spine and hip in patients with IGR and T2DM after adjusting for age and BMI. Although the OP detection rate was lowest in the T2DM group (7.47\%), Spearman's correlation analysis and rank transformation test results indicated that the correlation was not statistically significant after adjusting for age and BMI.

Previous studies of changes in BTMs in patients with diabetes have not provided consistent results. In a study by Starup-Linde et al, ${ }^{11}$ two kinds of BTMs and the bone markers such as OC and C-terminal cross-linked telopeptide (CTX) were found to be decreased in diabetic patients in comparison with nondiabetic controls among more than ten candidate markers.

The differing results in these studies may be related to differences in the patients' metabolism status, different levels of glucose control, and differing courses of DM. Our results indicated that concentrations of $\beta$-CTX, BGP, and P1NP were significantly different in the three glycometabolism groups after adjusting for age, $\mathrm{Cr}$, and BMI. However, multivariate statistical analysis showed that only BGP among the BTMs exhibited a slightly negative correlation with glucose metabolism, which was an independent influencing factor, although the effect was weak $(\mathrm{OR}=0.96)$.

$\mathrm{BGP}$ is considered to be a valuable marker for evaluating bone turnover and bone formation. The characteristics of $\mathrm{DM}$ and bone metabolism caused by old age are of the low conversion type. Although BGP was found to be influenced by abnormal glucose metabolism in this study, the correlation was a slightly negative one $(r=-0.08$; OR $=0.96)$. It shows a protective effect of BGP on DM. The direct effect of BGP on the $\beta$ cell stimulating insulin secretion and the indirect effect on stimulating secretion of adiponectin may explain the negative correlation. ${ }^{12}$ The hyperglycemia status may impair bone formation prevalently influencing bone activity directly by modifying BGP signaling pathways. This may be another reason for the negative correlation. ${ }^{13}$ Other clinical studies have suggested that BGP has a negative correlation with blood glucose, $\mathrm{HbA1c}$, and insulin resistance. Furthermore, multivariate analysis has revealed that BGP is an independent factor influencing blood glucose and HbAlc. ${ }^{14-16}$ Our study has shown that BGP is negatively correlated with FPG and PPG $(P<0.05)$. This finding is consistent with previous studies and reflects the relationship between BGP and energy metabolism.

25( $\mathrm{OH}) \mathrm{D}$ was positively correlated with the presence of abnormal glycometabolism $(r=0.08 ; P<0.01)$. Previous studies showed that vitamin D may contribute to the development of T2DM. Insulin secretion may be influenced by vitamin D indirectly for its role in the regulation of calcium flux through the cell membrane combined with its role in the synthesis and regulation of calbindin, which is a vitamin Ddependent Ca-binding protein in pancreatic $\beta$ cells. ${ }^{17}$

In this cross-sectional study, BMD was not significantly different between the T2DM and normal patient groups. Previous studies have reported that, while the BMD of patients with T2DM is normal or a little higher than that of nondiabetic controls, the fracture risk is increased in patients with T2DM. ${ }^{2,18,19}$ The reasons for the increased risk of osteoporotic fracture in diabetes are complex and include a decrease in bone mass, the macrovascular and microvascular complications of diabetes, the neurological effects of the disease, etc. As it is clear that the BMD of patients with diabetes does not really reflect bone fragility, further research is needed to assess differences in the fracture rate between diabetic and nondiabetic patients.

Our study has provided additional information for the association between T2DM and OP in men of China of Asian general population. BMD seems not to be higher in Chinese men as it does in other population. It has implications for future research in Asian.

Our study also has some limitations. The first limitation was that although we intended to recruit all the eligible subjects, not all of them accepted to join this study. There is a selection bias in this study. Although we have reduced the proportion of nonresponse rate as much as possible, this study population can represent the natural population of Beijing. The second limitation was that we did not collect the hip circumference data; for the routine BMD measurement data in China which contain height, weight, and so on, hip circumference was still not included. We will include hip circumference in later studies.

\section{Conclusion}

This study has shown that differences in BMD between patients with T2DM, IGR, and normal glucose metabolism 
are not statistically significant after adjusting for age and BMI. Among the various BTMs for which a relationship with abnormal glucose metabolism was evaluated, BGP was the only one to show a statistically significant correlation, albeit a slightly negative one.

\section{Acknowledgments}

Editorial assistance with the manuscript was provided by Content Ed Net, Shanghai Co. Ltd. Funding for this study was provided by the Chinese National High-Tech Research and Development Program (863 Program; no 2014AA022301).

\section{Disclosure}

The authors report no conflicts of interest in this work.

\section{References}

1. Xu Y, Wang L, He J. China Noncommunicable Disease Surveillance Group. prevalence and control of diabetes in Chinese adults. JAMA. 2013;310: 948-959.

2. Poiana $C$, Capatina C. Fracture risk assessment in patients with diabetes mellitus. J Clin Densitom. 2017;20(3):432-443.

3. Chen P, Li Z, Hu Y. Prevalence of osteoporosis in China: a meta-analysis and systematic review. BMC Public Health. 2016;16(1):1039.

4. Abdulameer SA, Sulaiman SA, Hassali MA, Subramaniam K, Sahib MN. Osteoporosis and type 2 diabetes mellitus: what do we know, and what we can do? Patient Prefer Adherence. 2012;6:435-448.

5. Conradie M, Conradie MM, Kidd M, Hough S. Bone density in black and white South African women: contribution of ethnicity, body weight and lifestyle. Arch Osteoporos. 2014;9:193.

6. Albright F. Osteoporosis. Ann Intern Med. 1947;27(6):861-882.
7. de Liefde II, van der Klift M, de Laet CEDH, van Daele PLA, Hofman A, Pols HAP. Bone mineral density and fracture risk in type-2 diabetes mellitus: the Rotterdam study. Osteoporos Int. 2005;16(12):1713-1720.

8. Majima T, Komatsu Y, Yamada T, et al. Decreased bone mineral density at the distal radius, but not at the lumbar spine or the femoral neck, in Japanese type 2 diabetic patients. Osteoporos Int. 2005;16(8):907-913.

9. Zhou Y, Li Y, Zhang D, Wang J, Yang H. Prevalence and predictors of osteopenia and osteoporosis in postmenopausal Chinese women with type 2 diabetes. Diabetes Res Clin Pract. 2010;90(3):261-269.

10. Ma L, Oei L, Jiang L, et al. Association between bone mineral density and type 2 diabetes mellitus: a meta-analysis of observational studies. Eur J Epidemiol. 2012;27(5):319-332.

11. Starup-Linde J, Eriksen SA, Lykkeboe S, Handberg A, Vestergaard P. Biochemical markers of bone turnover in diabetes patients - a metaanalysis, and a methodological study on the effects of glucose on bone markers. Osteoporos Int. 2014;25(6):1697-1708.

12. Patti A, Gennari L, Merlotti D, Dotta F, Nuti R. Endocrine actions of osteocalcin. Int J Endocrinol. 2013;2013(3):1-10.

13. Montagnani A, Gonnelli S. Antidiabetic therapy effects on bone metabolism and fracture risk. Diabetes Obes Metab. 2013;15(9):784-791.

14. Liu DM, Guo XZ, Tong HJ, et al. Association between osteocalcin and glucose metabolism: a meta-analysis. Osteoporos Int. 2015;26(12): 2823-2833.

15. González-García ZM, Kullo IJ, Coletta DK, Mandarino LJ, Shaibi GQ. Osteocalcin and type 2 diabetes risk in Latinos: a life course approach. Am J Hum Biol. 2015;27(6):859-861.

16. de Pergola G, Triggiani V, Bartolomeo N, et al. Independent relationship of osteocalcin circulating levels with obesity, type 2 diabetes, hypertension, and HDL cholesterol. Endocr Metab Immune Disord Drug Targets. 2016;16(4):270-275.

17. El-Fakhri N, Mcdevitt H, Shaikh MG, Halsey C, Ahmed SF. Vitamin D and its effects on glucose homeostasis, cardiovascular function and immune function. Horm Res Paediatr. 2014;81(6):363-378.

18. Dede AD, Tournis S, Dontas I, Trovas G. Type 2 diabetes mellitus and fracture risk. Metabolism. 2014;63(12):1480-1490.

19. Kurra S, Fink DA, Siris ES. Osteoporosis-associated fracture and diabetes. Endocrinol Metab Clin North Am. 2014;43(1):233-243.
Clinical Interventions in Aging

\section{Publish your work in this journal}

Clinical Interventions in Aging is an international, peer-reviewed journal focusing on evidence-based reports on the value or lack thereof of treatments intended to prevent or delay the onset of maladaptive correlates of aging in human beings. This journal is indexed on PubMed Central, MedLine,

\section{Dovepress}

CAS, Scopus and the Elsevier Bibliographic databases. The manuscript management system is completely online and includes a very quick and fair peer-review system, which is all easy to use. Visit http://www.dovepress. com/testimonials.php to read real quotes from published authors. 